Proceedings

\title{
Laser-Induced Thermography for Quantitative Detection of Cracks in Building Materials ${ }^{\dagger}$
}

\author{
Lorenzo Palombi ${ }^{1}$, Gabriele Inglese ${ }^{2}$, Valentina Raimondi ${ }^{1}$ and Roberto Olmi ${ }^{1, *}$ \\ 1 IFAC-CNR, 50019 Sesto Fiorentino, Italy; 1.palombi@ifac.cnr.it (L.P.), v.raimondi@ifac.cnr.it (V.R.) \\ 2 IAC-CNR, 50019 Sesto Fiorentino, Italy; gabriele@fi.iac.cnr.it (G.I.) \\ * Correspondence: r.olmi@ifac.cnr.it \\ + Presented at the 15th International Workshop on Advanced Infrared Technology and Applications \\ (AITA 2019), Florence, Italy, 17-19 September 2019.
}

Published: 23 September 2019

\begin{abstract}
Laser-induced thermography is a an active technique using a laser source to heat a very small area on a side of a crack in a building material. The presence of a crack is easily detected as a sharp change in the temperature due to its insulating nature, but no information about its depth is directly available from the thermal image. The method described in this paper uses a heuristic form of the temperature on the surface of the heated specimen, which is transformed to a two-dimensional distribution. Then, a relation is used (called $\beta$-tool) between the thermal gap across the crack and the unknown depth of the damage. The purpose is that of making it possible to distinguish between shallow and deep fractures (more than $15 \mathrm{~mm}$ deep).
\end{abstract}

Keywords: laser-spot thermography; non-destructive evaluation; cracks

\section{Introduction}

When dealing with masonry bridges, in particular those made of reinforced concrete, material fractures (cracks) are one of the most important damages to be considered for assessing the bridge integrity. A technique allowing to estimate the depth of a crack is highly desirable, because it would allow to discard shallow cracks and concentrate the inspection to deeper ones, an action impossible to conduct "by the naked eye".

This work has been developed in the framework of a research project aimed to set-up procedures involving several imaging techniques, such as photogrammetry, 3D scanning, fluorescence lidar and thermography, as a support for the diagnostics on railway bridges. In particular, here we present the results obtained using laser-induced (also called "laser-spot") thermography on selected specimens of brick and concrete.

The latter is an active technique employed for detecting the presence of cracks in metallic specimens [1,2], also called flying-spot camera or flying-spot laser thermography when the laser beam scans a surface in a more or less automatic way $[3,4]$. The technique consists in heating a very small area of a material by means of a laser source. A fracture, being air-filled, is almost thermally insulating. Therefore, if the laser illuminates a side of it, the resulting surface temperature, after a suitable time, is highly asymmetrical (Figure 1).

That is more evident if we plot the temperature on the symmetry axis of the laser spot, as shown in Figure 2. The crack depth can be estimated from the temperature on such an axis, measured at suitable times after turning OFF the laser source, by means of a mathematical model briefly described in the following section. 


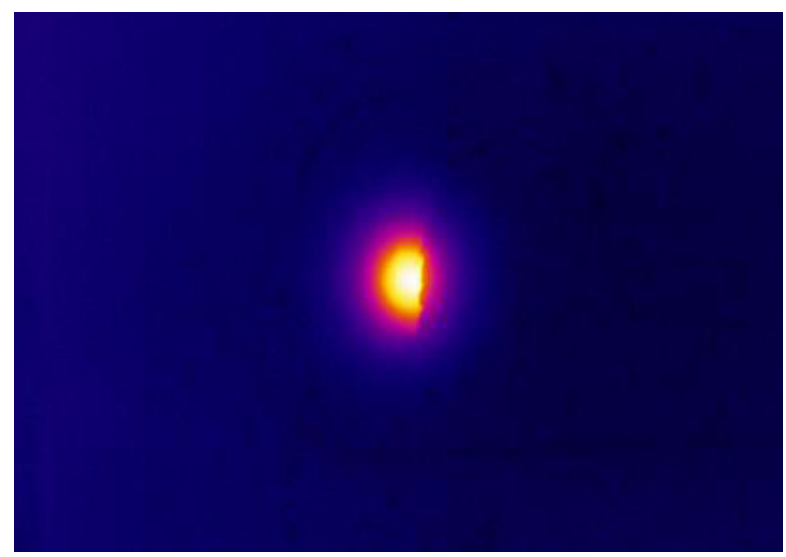

Figure 1. Temperature distribution around a crack, $20 \mathrm{~s}$ after laser OFF.

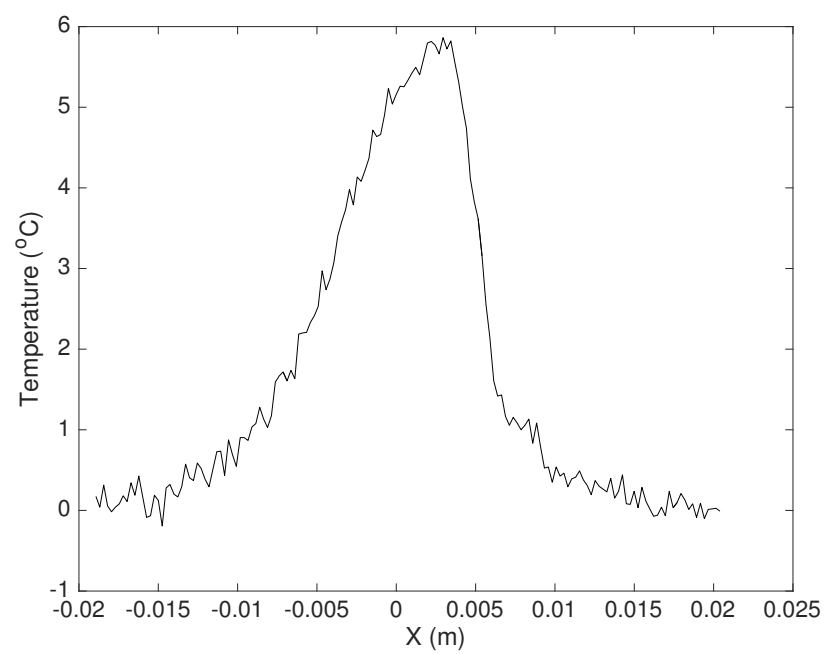

Figure 2. Temperature on the symmetry axis, $20 \mathrm{~s}$ after laser OFF.

\section{Results and Discussion}

Figure 1 shows a temperature map acquired $20 \mathrm{~s}$ after the laser source power OFF, by means of a thermo-camera FLIR T1020 equipped with the standard lens (focal length $36 \mathrm{~mm}$, FOV $28^{\circ} \times 21^{\circ}$. The laser source employed in the experiments is a pulsed laser working at wavelengths $532 \mathrm{~nm}$ and $1064 \mathrm{~nm}$, with an average energy of about $40 \mathrm{~mJ}$ per pulse (mix of the above two wavelengths). The pulse frequency was $20 \mathrm{~Hz}$, such to give an average output power of about $0.8 \mathrm{~W}$, corresponding to a power density of about $1.6 \mathrm{~W} / \mathrm{cm}^{2}$ at the target.

The procedure for determining the crack depth from the experimental data consists in: (1) transforming experimental data from 3D to 2D; (2) developing a 2D finite-element (FE) model of the specimen (using COMSOL Multiphysics ${ }^{\circledR}$ [5] and computing the $\beta$ - curve [6]; (3) using the temperature gap across the crack as an input to the $\beta$ - tool to obtain an estimation of the fracture depth.

The model is based on a theorem (Humps Theorem, HT) [7] establishing a relationship among the temperature values across the crack (temperature gap) and the temperature at the same points in absence of the crack (background temperature). From the HT, denoting by the subscripts " 1 " and " 2 " the temperatures at such points, we obtain the following relation among the temperature $u$ of the cracked specimen and the background temperature $v$ :

$$
u_{1}-u_{2}-\left(v_{1}-v_{2}\right) \approx \beta\left(v_{1}+v_{2}\right)
$$


The value of $\beta$ obtained from (1) is used as an input into the curve constructed by the FE model for regular, vertical cracks of given width $\left(u_{1}\right.$ and $u_{2}$ are experimental measured values). Figure 3 shows such a curve, computed varying the crack depth.

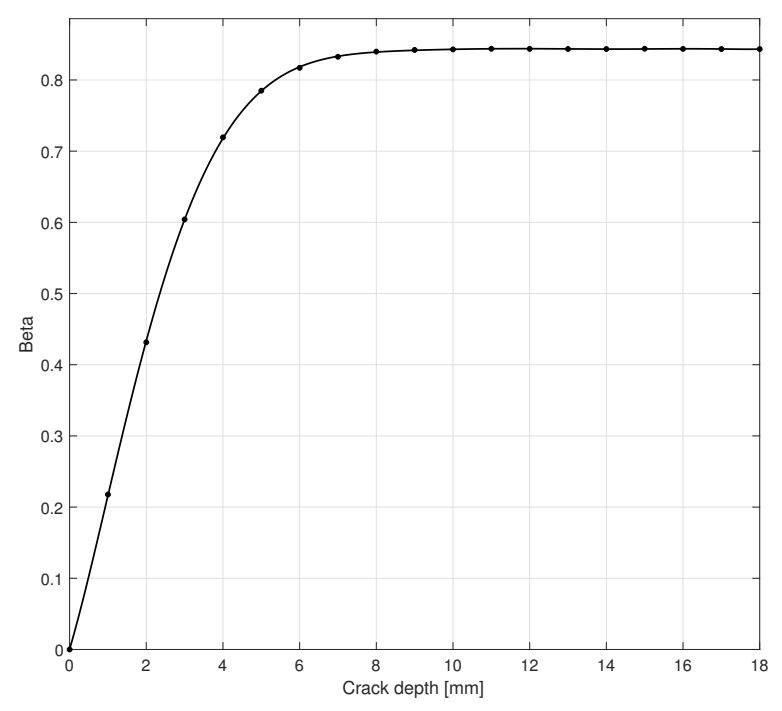

Figure 3. $\beta$-tool curve for the experiment.

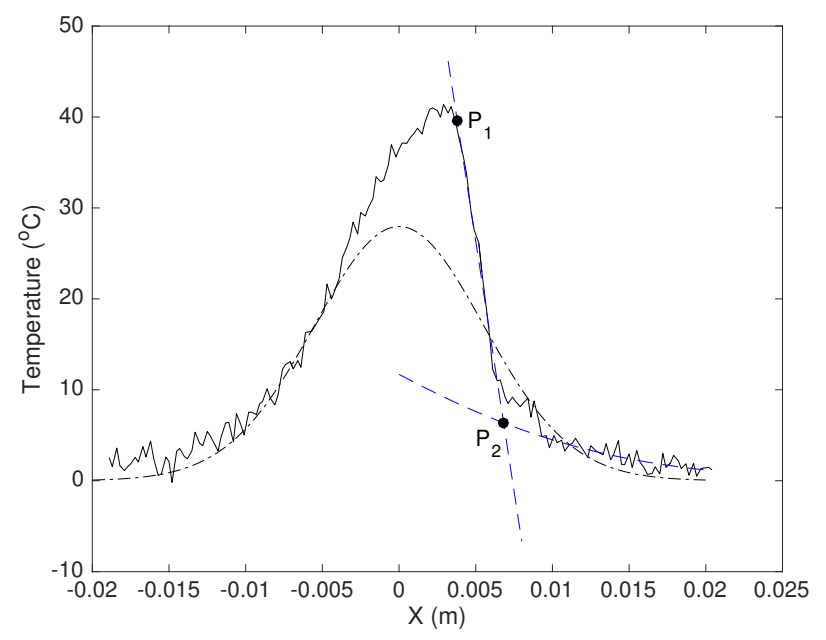

Figure 4. 2D Temperature across the crack (solid line) and background temperature (dash-dot line).

The construction of the $\beta$-curve requires a knowledge of the physical characteristics of the material under investigation, in particular its density, specific heat and thermal conductivity. An uncertainty in the true value of such parameters reflects on a systematic error on the crack depth obtained by the $\beta$-tool. However, the correctness of such parameters can be easily tested by comparing the measurements obtained on a sound part of the specimen with FE simulation results.

All the theoretical development assumes a two-dimensional geometry and, correspondingly, a 2D physics. Actual cracks are essentially two-dimensional, because their extension is usually very large compared to their width and to the laser spot size. Nevertheless, the shape of the laser spot on the specimen surface is far to be one-dimensional, as the mathematical model - assuming a continuous line-source - would require [6]. Instead of developing a three-dimensional model (feasible in principle, but computationally heavy), we prefer to transform 3D experimental data into "equivalent" 2D data. That is possible if we are interested in data belonging to the symmetry plane of the laser source, taken as the XZ plane of the 2D model. Referring to [6] for the details of the transformation procedure, the 
equivalent $2 \mathrm{D}$ temperature $U_{2}$ is obtained by the real 3D temperature $U_{3}$ by means of the Green's functions (source solutions) in the 2D and 3D cases, respectively.

$$
U_{2}(x, 0, t)=U_{3}(x, 0,0, t) \frac{\int_{-\epsilon}^{\epsilon} G_{2 c} d x^{\prime}}{\int_{-\epsilon}^{\epsilon} \int_{-\epsilon}^{\epsilon} G_{3 c} d x^{\prime} d y^{\prime}}
$$

Equation (2) comes from the relationship among the continuous-source Green's functions for 3D (point-source) and 2D (line-source) half-spaces, that for a homogenous medium (without cracks) are respectively:

$$
\begin{aligned}
G_{3 c} & \sim \frac{\operatorname{erfc}\left(-\frac{r}{2 \sqrt{\alpha t}}\right)}{2 \pi \alpha r} \\
G_{2 c} & \sim-\frac{\operatorname{Ei}\left(-\frac{\rho^{2}}{4 \alpha t}\right)}{2 \pi \alpha}
\end{aligned}
$$

where $\rho^{2}=\left(x-x^{\prime}\right)^{2}+z^{\prime 2}$ and $r^{2}=\left(x-x^{\prime}\right)^{2}+\left(y-y^{\prime}\right)^{2}+z^{\prime 2}$, with $G_{3 c}$ expressing the temperature on the plane $z=0$ due to a continuous point-source in $\left(x^{\prime}, y^{\prime}, z^{\prime}\right)$ and, analogously, $G_{2 c}$ expressing the temperature on the line $z=0$ due to a continuous line-source in $\left(x^{\prime}, z^{\prime}\right) . G_{3 c}$ and $G_{2 c}$ are expressed in terms of combinations of error functions and exponential-integral functions [6].

Assuming a "rectangular" laser source of width $2 \epsilon$ on the plane (line, in the 2D case) $z=0$, we can compute the temperatures $u_{3}(x, y, z, t)$ and $u_{2}(x, z, t)$, by convolving the Green's functions (3) and (4) with the laser power function $\phi\left(x^{\prime}, y^{\prime}\right)\left(\phi\left(x^{\prime}\right)\right.$ in the 2D case) which is located on the $z^{\prime}=0$. In particular, on the "accessible" surface $z=0$ :

$$
\begin{gathered}
u_{3}(x, y, t)=\frac{\phi_{0}}{\rho c} \int_{-\epsilon}^{\epsilon} \int_{-\epsilon}^{\epsilon} G_{3 c}\left(x, y, 0, t \mid x^{\prime}, y^{\prime}, 0\right) d x^{\prime} d y^{\prime} \\
u_{2}(x, t)=\frac{\phi_{0}}{\rho c} \int_{-\epsilon}^{\epsilon} G_{2 c}\left(x, 0, t \mid x^{\prime}, 0\right) d x^{\prime}
\end{gathered}
$$

The relationship (2), which is rigorously true in absence of the crack, eventually comes out assuming the approximate correctness of the $\beta$ model for the cracked 2D-region, on the $x$ axis ( $x_{0}$ being the crack abscissa):

$$
u_{\sigma 2}(x, 0, t)= \begin{cases}u_{0}(x, t)+\beta u_{0}\left(2 x_{0}-x, t\right) & x<x_{0} \\ (1-\beta) u_{0}(x, 0, t) & x>x_{0}\end{cases}
$$

relating the temperature $u_{\sigma}$ in the cracked case with the (background) temperature $u_{0}$ in the homogeneous case.

Figure 4 shows the equivalent 2D temperature computed from the data of Figure 2. A piecewise interpolation is performed, based on the following reasoning. We know that the temperature behavior immediately at the right of the crack edge should be a gaussian, and that the temperature drops off linearly across the crack. Therefore, the procedure consists in fitting a gaussian on the data immediately at the right of the temperature "anomaly" (discrepancy among model and experiment), and perform a linear fitting on the data across the crack opening, as shown in Figure 4. The crossing $P_{2}$ among those fitting curves identifies the right edge of the gap. Eventually, we are able to compute the $\beta$ value (1) in terms of the temperatures in $P_{1}$ and $P_{2}$, which is 0.71 , giving a depth of $4 \mathrm{~mm}$, slightly under-estimated with respect to the actual depth (measured by means of a thin paper sheet to be of the order of $5 \mathrm{~mm}$ ). Such an under-estimation is expected because real cracks usually shrink with depth, and the $\beta-$ tool model computes the depth of an equivalent constant-width fracture.

The whole procedure (3D-2D transformation of data; gaussian fitting; $\beta-$ tool) can be repeated at several measurement times, to obtain a more robust estimation of the crack depth. 
Funding: This research was funded by the Tuscany Region FAR-FIS research project "TOSCA-FI".

Conflicts of Interest: The authors declare no conflict of interest.

\section{References}

1. Li, T.L.; Almond, D.P.; Rees, D.A.S. Crack imaging by scanning pulsed laser spot thermography. NDT E Int. 2011, 44, 216-225.

2. Pech-May, N.W.; Oleaga, A.; Mendioroz, A.; Salazar, A. Fast Characterization of the Width of Vertical Cracks Using Pulsed Laser Spot Infrared Thermography. J. Nondestruct. Eval. 2016, 35, 22.

3. Schlichting, J.; Ziegler, M.; Maierhofer, C.; Kreutzbruck, M. Flying Laser Spot Thermography for the Fast Detection of Surface Breaking Cracks. In Proceedings of the 18th World Conference on Nondestructive Testing, Durban, South Africa, 16-20 April 2012.

4. Gruss, C.; Lepoutre, F.; Balegeas, D. Nondestructive evaluation using a flying-spot camera. In Proceedings of the 8th International THERMO Conference, Budapest, Hungary, 2-4 June 1993.

5. COMSOL Multiphysics ${ }^{\circledR}$, v. 5.2; COMSOL AB: Stockholm, Sweden, 2015. Available online: www.comsol.com.

6. Scalbi, A.; Olmi, R.; Inglese, G. Evaluation of fractures in a concrete slab by means of laser-spot thermography. Int. J. Heat Mass Transf. 2019, 141, 282-293

7. Inglese, G.; Olmi, R; Scalbi, A. Characterization of a vertical crack by means of local thermal analysis. arXiv 2019, arXiv:1905.10166.

(C) 2019 by the authors. Licensee MDPI, Basel, Switzerland. This article is an open access article distributed under the terms and conditions of the Creative Commons Attribution (CC BY) license (http:/ / creativecommons.org/licenses/by/4.0/). 